\title{
An Enterprise Resource Planning (ERP) Framework for Polytechnique Colleges in Ethiopia
}

\author{
Kassaye Tilahun, Patrick Cerna \\ Department of Information Technology, Federal TVET Institute, Addis Ababa, Ethiopia \\ Email address: \\ ktilahun92@gmail.com (K. Tilahun),pcerna@acm.org (P. Cerna) \\ To cite this article: \\ KassayeTilahun, Patrick Cerna. An Enterprise Resource Planning (ERP) Framework for Polytechnique Colleges in Ethiopia. Advances in \\ Wireless Communications and Networks. Vol. 3, No. 5, 2017, pp. 67-74. doi: 10.11648/j.awcn.20170305.13
}

Received: May 23, 2017; Accepted: June 23, 2017; Published: October 26, 2017

\begin{abstract}
Enterprise Resource Planning (ERP) is information system that integrates departments and functions across an organization into one computer system to streamline all the organizational process. Thus, each department across the organization shares a common centralized database, which enables the flow of information in the organization to be faster and reliable. Since, there is no common ERP framework design for specific domain; but most existing ERP framework design is based upon SOA (Service Oriented Architecture). This is because of SOA based ERP framework allows different applications to exchange data with one another effectively. This paper describes ERP framework design and implementation for Poly technique colleges in Ethiopia. To achieve this goal, we reviewed existing general ERP framework components and the process of various departments of poly technique colleges. The basic components we used in the framework are SOAP (Simple Object Access protocol) over HTTP (Hyper Text Transfer Protocol) protocol for communication between service consumers and service provider. The other component we applied is web service remote façade module integration mechanism to integrate each module. Finally, the researcher designed a novel framework that is suitable for the Poly Technique Colleges which show the integration among the internal department.
\end{abstract}

Keywords: ERP, SOA, Polytechnic, Framework, SOAP, Ethiopia

\section{Introduction}

In today's business world, companies are increasingly looking for flexibility, cost savings and efficiency in the business applications they acquire. The increased competition among companies has driven to look for new ways to succeed and remain competitive. In this IT led business environment, staying on the cutting edge of new technology acts as an enabler for companies to gain effectiveness and efficiency to face the competition.

The impact of Ethiopian TVET has been manifested in various sectors and national development agendas. In particular, tremendous achievements have been registered after the formulation of the new outcome based TVET Strategy in 2008. The Fourth Education Sector Development Plan (ESDP IV) in line with the Growth and Transformation Plan (GTP) has shown clear intention to ensure socioeconomic changes such as production of skilled manpower, poverty reduction, employment, technology transfer and the expansion of small-scale industries. Accordingly, a lot has been achieved in the TVET sector [1]. Nevertheless, there are still gaps to be filled that are attributed to quality and efficiency matters [2].

Ethiopia TVET is still accorded with limited financial support [3]. This is, obviously, because delivering high quality TVET is more expensive than general education. Firstly, TVET has lower teacher-student ratio as compared to general education. Secondly, TVET requires high investment cost for establishing facilities and supplying machinery and equipment. Thirdly, the cost for raw materials and consumables for delivering training on technical or hard occupations is high. Fourthly, high administrative cost is necessitated for the diversification and flexibility of occupational standards (OSs) and curricula. Lastly, the involvement of the private sector in TVET is limited to only low cost soft occupations and hence training delivery on the costly hard occupations is mostly left for the government [2]. The Ethiopian polytechnique and TVET college has nine core modules it divides into the following work process such as academic, technology transfer, finance, purchasing and 
property, human resource, internal audit, information communication plan and capacity building, public relation, and grievance handling.

Enterprise resource planning system is an attempt to create an integrated product that manages the majority of operations in a company. It integrates the various processes that are essential to running a business, including inventory and order management, accounting, human resources, customer relationship management (CRM) to create a single unified system rather than a group of separate, insular applications [4]. It also facilitates information sharing across organizational units and geographical locations.

The central feature of all ERP systems is a shared database that supports multiple functions used by different business units. In practice, this means that employees in different divisions for example, accounting and sales can rely on the same information for their specific needs [5]. Almost every organization whether an academic institution or a corporate companyuses ERP for managing various activities within the organization. For educational institutions, ERP is an information technology solution that integrates and automates recruitment, admissions, financial aid, student records, and most academic and administrative services.

The top reasons educational institution adopt ERP solutions is to replace legacy systems, improve customer service, and transform enterprise processes, modernize computer systems, improve administration, maintain competitiveness and increase operating efficiency [6].

\section{Related Works}

\subsection{ERP Definition}

Enterprise Resource Planning (ERP) is information system software that integrates departments and functions across an organization into one computer system. It runs off a single database, enabling various departments to share information and communicate with each other [6]. Integration is an extremely important part to ERP. Davenport claims that "ERP is an enterprise-wide information system, which facilitates the flow of information and coordinates all resources and activities within the organization". According to Deloitte [7] an ERP system is a packaged business software system that allows a company to: A.) Automate and integrate the majority of its business processes; B.) Share common data and practices across the entire enterprise; C.) Produce and access information in a real-time environment

Davenport [3] and Deloitte [4] ERP definitions focuses on the integration of various organizational networks, in particular functional divisions within the organizations like finance, human resource, registrar, department and inventory management while all departments are integrated to the unified system to streamlining the information they provide:"getting all the systems to talk to each other,". Shehabet al. [8] support Deloitte's and Davenport's perspective that they over-emphasize the integration aspect of ERP systems by asserting that ERP is more than a software package that seeks to integrate functional departments within an organization.

ERP's main goal is to integrate data and processes from all areas of an organization and unify it for easy access and flow of work. It aims to improve and streamline internal business processes, which typically requires reengineering of current business processes.

According to Yanet al [9] they defined ERP from four perspectives:

1) Business Process Perspective: ERP system as instruments for enabling enterprises to manage and streamline business processes, through crosses functional or cross-organizational integration.

2) Technological / Technical Perspective: ERP system as configurable, online real time interactive software package, which comprises of multiple modules (or applications) to support information processing function across the whole enterprises, through single database and uniform operating platform.

3) Communication Perspective: an ERP system as enterprise-wide information system that integrates all information flows and provides access to real-time information.

4) Functionality Perspective: ERP systems as an integrated set of programs that provide automations of various business procedures

\subsection{ERP Systems in Education}

Enterprise Resource Planning (ERP) is a business management system that integrates all component of the business with a view to facilitate the flow of information among all business functions. Although a large number of business organizations and higher education institutions abroad have adopted ERP. Many top western Universities are running their campus administration through ERP (Enterprise Resource Planning) systems. Universities are fundamentally different from business organizations in their decision making processes. When it comes to selecting and adopting an ERP system for universities, we need to ensure that it takes care of multiple perspectives pertaining to students, teachers, staff, administration, parents, and alumni. All the data is managed in a time sensitive manner along with the rules and policies applicable at that time, so whenever required, the exact information can be re-produced as it is. Various functions involving a number of campus requirements, Human Resources and Financials should be integrated.

Recently, many higher education institutions want to take advantages of ERP systems. They invest millions of dollars in ERP projects [9]. For example, the study conducted before six years tell us George town University in U.S.A spent nearly $\$ 60$ million on a campus wide ERP [10]. The University of Minnesota had a similar experience, when projected costs of $\$ 38$ million finally reached $\$ 60$ million. The investment in ERP systems represents the biggest investment in ICT (Information Communication Technology) for higher education institutions [11]. The top reasons 
institutes are adopting ERP solutions are to replace legacy systems, improve customer service, transform enterprise processes, improve administration, maintain competitiveness, increase operating efficiency [4]. The main advantages of ERP for higher education institutions are, according to ECAR organization (EDUCAUSE Center for Applied Research) are the following [12]:

a) Improved information access for planning and managing the institution.

b) Improved services for the faculty, students and employees.

c) Lower business risks.

d) Increased income and decreased expenses due to improved efficiency.

\subsection{ERP in Case of Australia Higher Education}

Australian universities have been under more pressures as a result of increasing number of students and increased government pressures to reform their structures, lower their costs and achieve greater administrative efficiency [9]. The Australian government has pressured universities into acting more like businesses, as the level of funding has significantly reduced [6]. However, one of the reasons that universities have implemented ERP systems is to improve performance and learning services, and also to become more efficient in their operations[13]. ERP systems have become a standard feature of most Australian higher education institutions and more than $85 \%$ of Australian universities have implemented ERP systems [12].

\subsection{ERP in Case of Romanian Higher Education}

Romanian universities have been choosing information systems based on the need to satisfy different requests in a short time. The universities have been forced to use in house developed information systems or to buy software solutions from small vendors having no experience in the field because of budget restrictions. These information systems have a low integration level and they are not based on homogeneous framework standards. They increase the proliferation of heterogeneous systems, complicating the integration among the various applications. Each application has its data and functions separated from the other applications already existing in the organization. These applications don't provide an integrated view of all activities in a university [6]. Current information systems have not been developed for strategic analysis and don't store historical data about students, courses and personnel. It is therefore impossible to develop a complex analysis that provides real time reports and useful indicators to the university management [6].

\subsection{ERP in Case of Indian Higher Education}

The introduction of the ERP in higher education institution in India has resulted in yet a further layer of change in universities to replace old administrative and management systems with new ones [14]. However, one of the reasons that universities have implemented ERP systems is to improve performance and learning services, and also to become more efficient in their operations, in part to deal with the range of other changes they have been facing. ERP system for Indian higher education developed in the direction of support student administration (student enrolment procedures, student enrolment, and student data), human resource management (monitoring of employees) and finance (accounting, payments, investments and budget).

Consequently, Indian higher education began to implement ERP systems to replace old and out dated systems with more efficient ones. In the process, ERP systems have become a standard feature of most Indian higher education institutions. Indian higher education institutions that have invested in ERP systems, the magnitude of the implementation project easily become the biggest information system project in the organization's history [14].

\subsection{ERP in Other Context}

Rani [15] conducted a study the benefits and impacts provided by ERP systems in higher education institution. Their study analyzes what role the ERP system plays in educational organizations and how it is helpful for them. ERP systems are considered to be the largest software applications adopted by most of the educational organizations along with some investments in their implementation. However, less research activities have been done regarding the ERP systems in a university environment. An increasing number of higher education institutions have introduced ERP systems in order to improve their operations and make them manageable and more transparent. ERP vendors are also aware of this fact, which is the reason they have already expanded their solutions in order to satisfy the needs of higher education institutions [15].

Similarly, Karia and Soliman [16] studied the benefits, challenges and issues of Enterprise Resource Planning (ERP) Systems in the Egyptian Higher Education Institutions. One of the challenges is that ERP system is a dynamic and complex large integrated packaged solution, therefore, it may create problems for the IT staff and management during the implementation phase, even, for those who might have a detailed understanding of their own organization. This may be due to the fact that universities possess an expanded array of systems; many of which may have competing functions whenever there are a specific needs. In the worst case scenario, universities may not have IT staff or management who are sufficiently well-trained to understand the complexities of organizational functions [16]. As the Egyptian HEIs are being met with significant challenges, the system of ERP introduces new techniques and tools that can provide solutions to the problems confronting Egypt's HEIs. The ERP characteristics and functions or modules leads to enhance HEIs operational performance along with improving the quality of educational services provided. Future research is suggested to examine the impact of ERP system on HEI's performance [16].

On other hand, Singh [17] conducted a research on Implementation of ERP education in postgraduate accounting 
information systems (AIS) course. The primary goal of the study was to introduce postgraduate AIS students to a realworld ERP environment. Students completed an in-class survey on their experiences in using SAP ERP over three semesters (Semester 2, 2013 to Semester 2, 2014). The instrument consisted of 4 questions ranked on a 5 point Likert scale, and one open-ended question inviting additional comments. There were 147 students enrolled in the course during this period. One-hundred and thirty five (135) responses were received, representing a response rate of $91.8 \%$. The results of the survey were used to determine the appropriateness of including SAP ERP education in the course [18]. The study concludes that conclude that learning hands-on ERP skills is important and very relevant for a student's career. Student experiences show that this approach provides them with a practical real world view of information flows within an organization. The course integrated theoretical accounting and business concepts with a hands-on practical ERP component. Students had the opportunity to examine an organization from different functional aspects, while remaining aware of the organization as a whole [18].

Alternatively, Monwani [18] studied the impact ERP implementation process on User's performance. It was conducted in manufacturing organizations with a unique organizational culture, the results might not hold true in other organizations and environments of other cities. The period of comfort with the technology, level of experience, amount of usage and frequency of assessing ERP could be considered in order to segregate the ERP user into early adopter, late adopter or laggard. The study provides managers with a clear view of the relative performance of the various parts of the enterprise, which can be used to identify needed improvements and take advantage accordingly. Managers could periodically evaluate the performance indicators in the study, benchmark the results with the expected satisfaction levels and diagnose which factors are problematic and need further consideration [18].

Jinno [19] present the results of an analysis of implementing enterprise resource planning (ERP) effectiveness from the perspective of implementation policy and operational effectiveness. The results of this study, in terms of deciding to customize the policy and promoting its introduction to ascertain how much the business is affected it can be said that the best policy in enhancing the overall effect is ERP. However, just by throwing away existing business processes, including the processes that are the source of superiority, and making ERP implementation IT-focused, it is difficult to achieve effectiveness of ERP implementation [19].

\section{Existing and Proposed ERP Framework and Architecture}

To understand the existing ERP architecture, similar works on the area have been assessed. The architecture used is three-tier architecture; however, most of the existing ERP framework is based upon three-tier architecture. Huang [10] defined software architecture as the structure of a system that comprises software elements, their external visible characteristics, and the relationship between them. Lin [20] defines software architecture as "the fundamental organization of a system embodied in its components, their relationship to each other's and the environment, and the principles guiding its design and evolution". Jones defined architecture as the structure that is composed of components and rules that establish the basis for the interaction between them. All the definitions agree that architecture is concerned with the constituting parts of a system and the relationship between them [21].

While reviewing related work on ERP architecture, most ERP vendors like SAP R/3, Oracle and Microsoft Dynamics works based on three-tier architecture. They are presented in the following sections.

\subsection{Case of $S A P R / 3$}

SAP ERP, known as SAP R/3, is another prominent solution in the market. It is primarily based on a three-tier architectural style: the presentation layer, the application layer, and the database layer. The presentation layer represents a tiny application, namely sapgui.exe that is usually installed on the client's machine [22].

SAP R/3 is based on a three tier client/server technology. It is made up of three functionally distinct layers namely [22]:

The presentation layer: It provides a graphical user interface (GUI) that is connected with the application servers through a local (LAN) or a wide-area network (WAN).

The application layer: It comprises the business administration and responsible for processing of pre-defined and user-defined application programs.

The database layer:Implemented on top of a commercial database product that stores all data of the system.

Internet Communication Manager (ICM)

The Internet Communication Manager guarantees communication between the SAP System (SAP Web Application Server) andthe outside world via theprotocol HTTP, HTTPS and SMTP. In its role as a server the ICM can process requests from the Internet that arrive as Uniform Resource Locator (URL) with the server/port combination that the ICM can listen to.

\section{Software Deployment Manager (SDM)}

The Software Deployment Manager (SDM) is the standard tool that you use to install J2EE components on the AS (application Server) Java. The SDM is a client/serverapplication. The SDM Server runs on the AS Java side. This server is started automatically with the AS Java. A graphicaluserinterfaceisavailableas a client.

Figure 1 shows the 3-tier architecture of SAP R/3 consist of the presentation server, application server and database server. The presentation server is actually a program named sapgui.exe. It is usually installed on a user's workstation. To start it, the user double-clicks on an icon on the desktop or chooses a menu path. An application server is a set of executables that collectively interpret the ABAP/4 programs 
and manage the input and output for them. The database server is a set of executables that accept database requests from the application server. These requests are passed on to the RDBMS (Relation Database Management System) [22].

\subsection{Case of Oracle}

Oracle ERP is a three-tier system that is composed of four basic modules namely: Human Resources, Project Management, Finance, and Asset Management. These modules are built on top of a unified Oracle database. The interaction between these modules is achieved via the Business Event System (BES) that triggers message creation or consumption of any registered parties. Oracle currently offers an additional package, namely the SOA (Service Oriented Architecture) suite, which can be integrated with the E-Business suite in order to promote enhanced scalability. ERP applications can therefore be exposed on the Oracle Service Bus (OSB) as services. Recently, the key features of the SOA suite became an integral part of the Oracle Ebusiness suite R12.1 package with the inclusion of the Oracle EBS adapter However; these added features are sold with different licenses, which can be very expensive to some organizations [23].

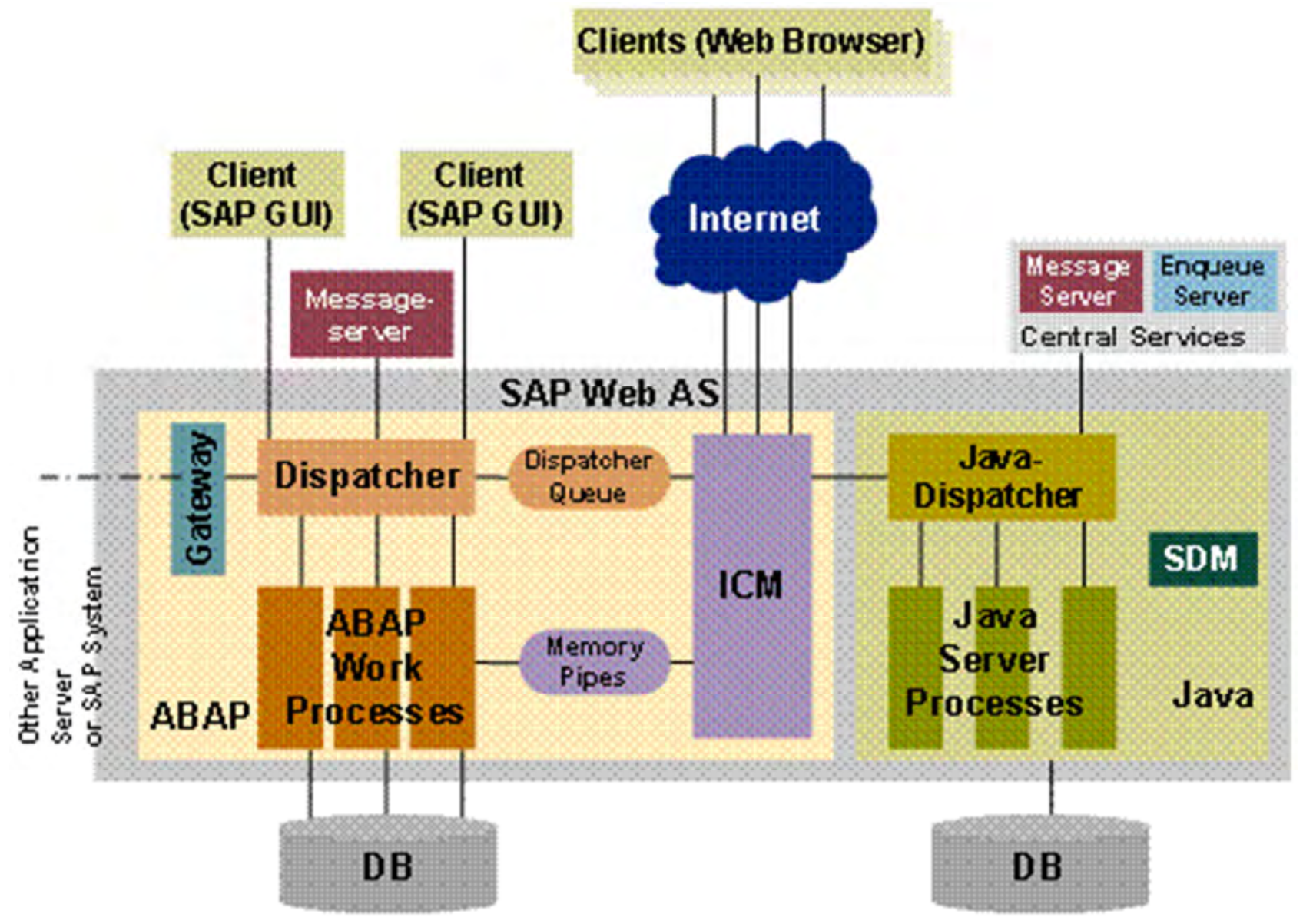

Figure 1. Three-tier architecture for $S A P R / 3$.

\subsection{Case of Microsoft Dynamics}

Microsoft offers a number of ERP solutions to suit various customer needs. One of the ERP solution that is known as a comprehensive solution is Dynamics AX [5]. It employs the three-tier architectural pattern, namely: client tier, Application Object Server (AOS) tier, and database tier. The client contains forms and reports code. AOS is used to execute application objects such as classes and queries. The database is normally used to store data for the ERP. Microsoft Dynamics AX utilizes the Application Integration Framework (AIF) to facilitate the integration of applicationto-application and also business-to-business. AIF supports the creation of generic web services and also document services; it also facilitates the consumption of external web services from within Dynamic AX [24].

Another ERP solution provided by Microsoft is the Dynamics GP, which is also based on a three-tier architectural pattern. The application tier is composed of three main components: the Dexterity tool and runtime, Dynamics Application Dictionary, and SQL server. The Dexterity tool is used to build the forms and also to attach scripting code using sanScript to applications. The Dexterity runtime environment is used to enable the execution of a functioning application to end-users. This tool is therefore responsible for the development and the execution of the application interfaces [24]. 
The Dynamics Application Dictionary (DAD) is responsible for storing the business logics in common component architecture such as COM + and DCOM [9], so other distributed applications can use them as service providers. The main design consideration of this dictionary is to separate the presentation logic from the actual business logic of an application, so services can be accessed independently of any form or application of the presentation layer [24].

\subsection{Proposed ERP Framework}

The researcher proposed a novel ERP framework design for poly technique colleges and the basic components of the framework are depicted on the Figure 2 as shown below.

Client

The client attribute includes different nodes such as operating system, connectivity constraint, GUI (Graphical
User Interface) and division of responsibility. The operating system includes the type of operating system supported by client software. The connectivity constraints check the types of networks that can connect clients to application servers.

Web Service

A Web Service is a software system designed to support interoperable machine to-machine interactionover a network and those services are autonomous components that process well-defined XML messages. It conform the following standard.

Extensible Markup Language (XML): is the basic building block of web services. All technologies used in web services are build on XML. An XML can be described as a metalanguage used to describe the format of other languages. It is a set of rules through which one can describe XML markup languages.

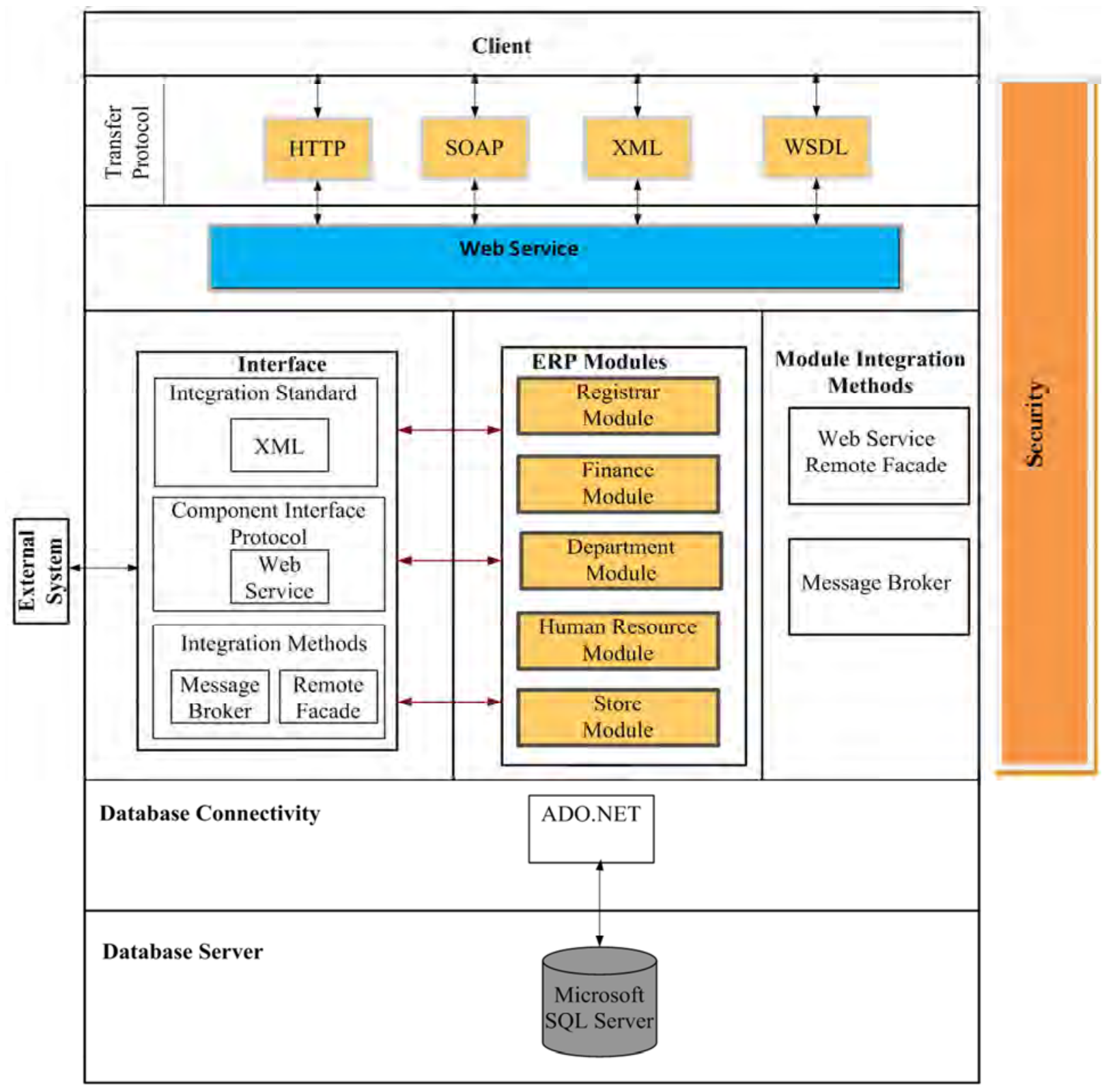

Figure 2. Proposed ERP Framework. 
1) Hypertext Transfer Protocol (HTTP): The protocol of the Internet is used by web services to send and receive message.

2) Universal Description, Discovery and Integration (UDDI): is XML based registry database that an XML provider could use to describe the web services and allows users to locate a web service.

ERP Module Integration Techniques

The proposed framework contains basic module such as: Finance, Department, Registrar, Human Resource and Store. There are two types of module integration technique using web service remote façade and message broker.

Message Broker

Message broker is a component that acts as an intermediary between the integrating modules. Using this method each module does not directly invoke each other but communicate only with the message broker. With each communication, message broker is provided with the logical identification of the destination module where the message broker delivers the message to that designated receiver. In a typical implementation, all modules need to get registered with the message broker prior to operation.

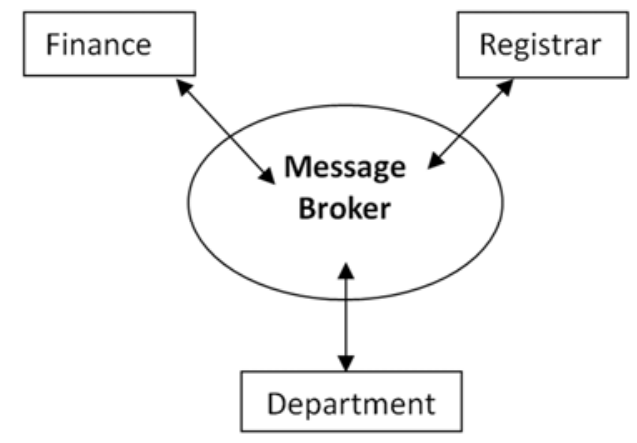

Figure 3. Message Broker Module Integration Method.

The drawback of message broker module integration is the introduction of a single point of failure the system will stop working the entire module around the message broker will stop communication.

\section{Web Service Remote Façade}

Web services provide a platform neutral approach for integration using standard XML protocols. These methods are based on open standards such as SOAP, WSDL and HTTP and therefore widely adapted unlike proprietary standards. Message broker integrate the module as centralized manner where as web service facades provide integration between ERP modules.

There are two main components exists in web service remote façade

1) Service Broker: This components act as the intermediary between modules and web services. The broker bundle the web reference with services and distribute the web services.

2) Web Service: This is the remote façade component that makes functionality remotely available. A web service use of SOAP over HTTP.

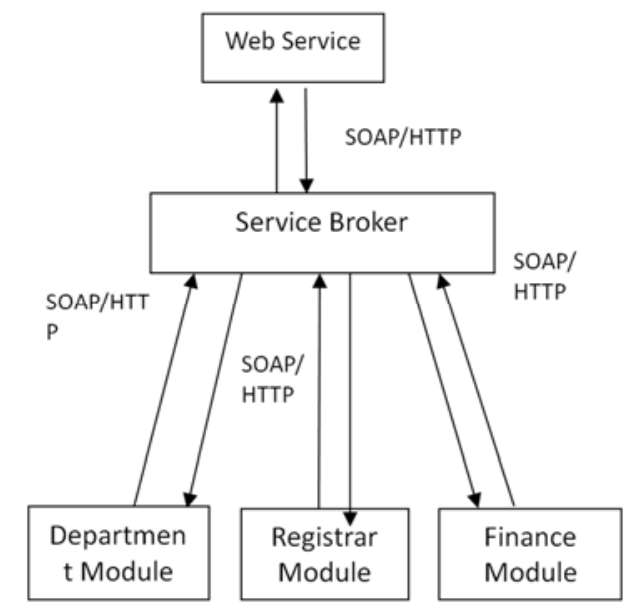

Figure 4. Web Service Remote Façade Module Integration Method.

Generally, we discussed two types of module integration method, however, for this project implementation we select web service remote façade module integration method because of the following reasons:

1) Message broker is off-the-shelf integration techniques but web service is based upon open standards.

2) A single point failure of the message broker the integration will halt the system. In case of web services integration does not introduced a single point failure.

Interface

The interface consists of three components such as Integration standard, component interface protocol and integration method that allows integrating the ERP system with the external environment. These listed interface type consists sub-component for instance the integration standard contain XML, component interface protocol according to this proposed framework it uses web service and integration method involved either using message broker or web service remote facade.

Database connectivity

The Database connectivity contains different components such as JDBC, OLBE and ADO.NET. Each of connectivity differs from each other depending on the Data base Management System (DBMS) used. For instance, the proposed ERP framework will use ADO.NET data base connectivity to access the SQL server.

Security

It consists of the following components:-

1) Identification and authentication - The first responsibility of information security within an ERP system is to ensure that legitimate and authorized user only access the ERP system.

2) Authorization - One of the most critical aspects to consider within ERP security is to restrict the access rights and actions of the users within the ERP system. The access rights of a user are controlled by the authority assigned to the user ID

3) Confidentiality - Protecting the confidentiality of data implies the assurance that only authorized people are able to view specific data sets. 
4) Integrity - Integrity means that only authorized users can modify the data of the ERP system. Modification refers to the update, deletion and creation of data within the ERP system.

\section{Conclusion}

Enterprise Resource Planning (ERP) is information system that integrates departments and functions across an organization into one computer system. It runs off a single database, enabling various departments to share information and communicate with each other. Based on the papers we review most existing ERP framework used Service Oriented Architecture for module integration it allows the ERP system integrates all information of different units using different platforms into one central database. The benefits of this approach include cost savings, improved business agility by decreasing time to deliver results, and enhanced collaboration and sharing of resource.

The researcherhas proposed a design ERP framework for Poly Technique colleges based on the existing general Service Oriented Architecture ERP framework. Since Service Oriented Architecture based ERP framework consists different components such as web service, Common Object Request Broker Architecture and Enterprise Java Beans. This ERP framework design focus on Service Oriented architecture using web service by the use of Simple Object Access protocol over Hyper Text Transfer Protocol and integrate the internal department of poly Technique College through web service remote façade. As a future work ERP framework design can be extended to support integration with other external organization, customization based on the business needs of poly technique colleges and add different plug in modules.

\section{References}

[1] MoE (2008) National Technical and Vocational Education and Training Strategy. 2nd edition Addis Ababa: (MoE).

[2] MoE(2010). TVET Leaders' and Trainers' Qualifications Framework (TLTQF). Addis Ababa.

[3] MoE(2010) Co-Operative and in Company Training hand book. Addis Ababa Ministry of Education.

[4] Alshawi, S., Themistocleous, M., and Almadani, R. (2004). "Integrating diverse ERP systems: a case study",Journal of Enterprise Information Management, Vol. 17 Issue: 6, pp.454462,https://doi.org/10.1108/17410390410566742

[5] Bingi, Sharma, Godla, K. (2004). Critical Issues Affecting an ERP Implementation. Information Systems Management, 5-12.

[6] Davenport, T. H. (2007). Putting the enterprise into enterprise systems. Harvard Business Review, July-August, 221-131.

[7] Deloitte. (2015). Your guide to a successful ERP journey. Deloitte. Com, 1-20.

[8] Shehab, E., Sharp, M., Supramaniam, L., and Spedding, T. 2009. Enterprise Resouce Planning: An Integrate Review. Business Process Management Journal. 10 (4) pp. 359-386.
[9] Yan, X., Rahmati, N., \& Lee, V. C. S. (2008). A review of literature on enterprise resource planning systems. Paper presented at the 5th International Conference on Service Systems and Service Management, Melbourne, Australia.

[10] Huang, Z. P. (2011). ERP implementation issues in advanced and developing countries. Jornal of Business Process Management, 221-276.

[11] Bologa, R. and Bologa A. R. (2011). Management Information Systems in RomanianUniversities. International Conference on e-Business, 425-428.

[12] Liu, S. W. (2000). ERP Strategic Investigation Research. Data control AcadamicResearch Discussion Thesis,Kaohsiung chungshan university,Taiwan., 99-120.

[13] Chu, H. L. (2002). ERP Crucial Success Factors (CSF) and Failure Causes Investigation. Electronic Business and Digital Lifestyle Discussion Panel, Taiwan Electronics Busines institute, Shita University Data Control Department., 56-78.

[14] Ifenedo, P. (2011). Interactions between organizational size, culture, and structure and some IT factors in the context of ERP success assessment: an exploratory investigation. Journal of Computing Information Systems, 28-47.

[15] Rani, S. (2016). A Review of ERP Implementation in Higher Education Institutions. International Journal of Advanced Research in Computer Science and Software Engineering, 3(10), 572-576.

[16] Karia, N. and Soliman, M. (2016). Enterprise Resource Planning ( ERP ) Systems in the Egyptian Higher Education Institutions: Benefits, Challenges and Issues. International Conference on Industrial Engineering and Operations Management. 1935-1943.

[17] Singh, K. (2016). Implementing enterprise resource planning education in a postgraduate accounting information systems course, 8(1), 27-37.

[18] Motwani, B. (2015). Impact of Enterprise Resource Planning (ERP) Implementation Process on Users Performance. Anvesha, 8(1), 44-56.

[19] Jinno, H., Abe, H., \& Iizuka, K. (2017). Consideration of ERP effectiveness: From the perspective of ERP implementation policy and operational effectiveness. Information (Switzerland), 8(1). https://doi.org/10.3390/info8010014

[20] Lin, F. 1. (2010). Research Regarding Relationship between ERP Package Software strategy target Strategic Targets and Key Factors. Taiwan University Business masters Thesis, Taiwan., 98-187.

[21] Cornford, J. and Pollock N. (2001). Cornford, J. and N. Pollock. Customizing Industry Standard Computer, 45-67.

[22] SAP Brains Online. SAP R3 Architecture Introduction. Retrieved from http://sapbrainsonline.com/help/sap-r3architecture-introduction.html.Accessed on Nov. 2016.

[23] Alkazemi, B. Y. (2012). A conceptual framework to analyze enterprise business solutions from a software architecture perspective. International Journal of Computer Science Issues, 9(3), 77-84.

[24] Microsoft Online. Microsoft Dynamics ERP and Business Software. Retreived from https://www.microsoft.com/enus/dynamics365/home.Accessed on Nov. 2016. 